\title{
El Impacto de la Cirugía de Mastectomía en la Satisfacción Sexual en Mujeres con Pareja
}

\author{
Mastectomy Surgery's Impact on the Sexual Satisfaction of Women with Sexual \\ Partners
}

Paola M. Buffit Torres ${ }^{1 *}$, Caleb Esteban ${ }^{2}$, Eida M. Castro ${ }^{3} \&$ Astrid Irizarry-Rodríguez 4

1 Ponce Health Sciences University, Ponce, Puerto Rico. (D) http://orcid.org/0000-0001-8370-5564

2 Ponce Health Sciences University, Ponce, Puerto Rico. (D) https://orcid.org/0000-0002-0960-6311

3 Ponce Health Sciences University, Ponce, Puerto Rico. (D) https://orcid.org/0000-0003-3889-9114

4 Ponce Health Sciences University, Ponce, Puerto Rico. (D) https://orcid.org/0000-0002-2298-7323

* Correspondencia: paolapsyd@gmail.com

Recibido: 13 abril 2021 | Aceptado: 29 julio 2021 | Publicado: 13 agosto 2021

\section{WWW.REVISTACARIBENADEPSICOLOGIA.COM}

\section{Citar como:}

Buffit Torres, P. M., Esteban, C., Castro, E. M., \& Irizarry-Rodríguez, A. (2021). El impacto de la cirugía de mastectomía en la satisfacción sexual en mujeres con pareja. Revista Caribeña de Psicología, 5, e5537.

https://doi.org/10.37226/rcp.v5i1.5537

\section{RESUMEN}

El cáncer de mama es el segundo tipo de cáncer más frecuente entre las mujeres de los Estados Unidos y Puerto Rico. La mastectomía es uno de los diversos tratamientos para el cáncer de mama. En Puerto Rico, son escasas las investigaciones en el estudio del impacto de la mastectomía en la satisfacción sexual y en la relación de pareja. Los objetivos de este estudio fueron: (a) evaluar si la cirugía de mastectomía impacta significativamente la satisfacción sexual de las participantes, (b) evaluar si la cirugía de mastectomía impacta la relación de pareja de las participantes, (c) evaluar si la cirugía de mastectomía impacta los síntomas de depresión de las participantes. Se llevó a cabo un estudio de método cuantitativo de tipo exploratorio con una muestra de 29 mujeres con cáncer del mama y 30 mujeres sin cáncer como grupo comparativo. Se utilizó un cuestionario sociodemográfico y sexual, el ESSS, el ESP10 y el PHQ-8. Se llevó a cabo un análisis de prueba U de Mann-Whitney de muestras independientes para la comparación de los grupos. Los resultados sugieren que la muestra de mujeres con cáncer de seno sometidas a mastectomía reportó significativamente menor satisfacción sexual y satisfacción en la relación de pareja, que las participantes del grupo comparativo. No se encontró diferencias significativas en los síntomas de depresión. Además, se encontraron diferencias significativas para las variables de edad y tiempo en la relación. Estos resultados exponen la importancia de que el tratamiento para las mujeres con cáncer de seno y sometidas a mastectomía sea integrado desde un enfoque interdisciplinario.

Palabras Claves: cáncer de mama, impacto psicológico del cáncer, satisfacción sexual, sexualidad femenina, relación de pareja 


\begin{abstract}
Breast cancer is the second most common type of cancer among women in the United States and Puerto Rico. Mastectomy is one of several treatments for breast cancer. In Puerto Rico, research is scarce in the study of the impact of mastectomy on sexual satisfaction and relationships. The objectives of this study were to: (a) assess whether mastectomy surgery significantly impacts sexual satisfaction of the participants; (b) assess whether mastectomy surgery impacts the relationship of the participants; (c) assess is there were relations between the sociodemographic data and the principal variables. An experimental quantitative study was conducted with a sample of 29 women with breast cancer and 30 women without cancer for a comparative group. A sociodemographic and sexual questionnaire, ESSS, ESP-10, and PHQ-8 were administered. A Mann-Whitney U test analysis was performed for groups comparison. The results suggest that the sample of women with breast cancer undergoing mastectomy reported less sexual and relationship satisfaction, compared with the comparative group. Significant differences in depression symptoms were not founded. In addition, significant differences were found for age and time variables in the relationship. These results set out the importance of treatment for women with breast cancer and undergoing mastectomy to be integrated from an interdisciplinary approach.
\end{abstract}

Keywords: breast cancer, cancer psychological impact, sexual satisfaction, women's sexuality, relationships

\section{INTRODUCCIÓN}

Se estima que una de cada ocho mujeres puede desarrollar cáncer de mama invasivo a lo largo de su vida (Instituto Nacional del Cáncer, 2015). Para combatir esta patología existen diferentes tipos de tratamiento entre los que se encuentra la cirugía de mastectomía. Desde una perspectiva vivencial, es imprescindible destacar que el diagnóstico de cáncer de mama conlleva un impacto emocional relacionado con la posible muerte de la paciente. No obstante, según Vázquez-Ortiz et al. (2010), el ámbito emocional no es el único afectado por la enfermedad. Debe tenerse en cuenta que las secuelas psicológicas de la mastectomía y los tratamientos contra esta patología afectan directamente a la autoestima, la identidad femenina, la imagen corporal y las relaciones afectivosexuales de la mujer que padece la enfermedad.

\section{Impacto de la Falta del Seno (Mama) en su Autoima- gen y Autoconcepto}

A la mama o el seno están asociadas muchas representaciones como capacidad de mantener la vida a través de la alimentación (lactancia y la participación dentro de las relaciones sexuales como zona erógena o elemento integrante de la excitación sexual. Los efectos de la cirugía y de los tratamientos implican pérdidas y modificaciones visibles de aspectos físicos que a nivel social pueden definir el concepto de femineidad. La mastectomía se percibe como un tipo de ataque a la feminidad, dado que esta se define como el conjunto de características que identifican a una mujer, incluidas las físicas. Para los latinos, el pecho en particular está asociado con la sensualidad y la fertilidad. Hungr et al. (2017) realizaron estudios cualitativos de sobrevivientes de cáncer de mama hispanas/latinas. Dichos estudios proporcionan ejemplos claros de cómo, con base en experiencias negativas muy personales de sus senos alterados después del cáncer, las mujeres perciben: (1) una pérdida en el poder femenino y (2) una pérdida en su capacidad para atraer a un compañero y sentirse atractivas (Hungr et al., 2017).

\section{Comparativas de la Autoimagen antes y Después del Tratamiento}

Estudios recientes han señalado que la primera vez que una mujer se enfrenta a la nueva imagen de su cuerpo después de la operación percibe una alteración de la imagen corporal de manera inmediata, esto conlleva una serie de significados, percepciones y sensaciones respecto a su nueva imagen (Freysteinson et al., 2015). Estos autores también indicaron en su estudio que las reacciones ante este evento envuelven preocupaciones sobre la aceptación de su pareja hacia su nuevo pecho y sobre cómo podrán trabajar su nueva imagen a nivel visual.

En un estudio realizado por Kocan y Gursoy (2016), algunas mujeres reportaron sentirse mal, asustadas, extrañas y diferentes, debido a que describen la mastectomía como un evento difícil y perturbador. Otras mujeres señalaron que ese primer vistazo a la incisión quirúrgica las sorprendió y les causó 
reacciones emocionales mixtas. Por otra parte, aquellas mujeres ya han experimentado una reconstrucción física de sus senos, señalan que, si bien esta disminuye la angustia de la pérdida, en ellas persiste un vacío, debido a que probablemente en su psique emerjan nuevas metáforas en torno a la enfermedad y el pecho reconstruido. No obstante, las mujeres sometidas a la reconstrucción mamaria tienen una mejor imagen corporal. La reconstrucción mamaria permite a las mujeres pasar de su experiencia de "persona enferma" y en tratamiento del cáncer a poder administrar mejor su imagen y potencialmente reducir la ansiedad por el miedo a la recurrencia (Mckean et al., 2013). Además, la reconstrucción puede ayudarlas a ser discretas acerca de su tratamiento, así como reanudar la vida familiar, permitiéndoles mantener una apariencia normal y ejercer cierto control sobre cómo afrontan su enfermedad y el tratamiento (Sontag, 2006).

\section{Sexualidad después de la Mastectomía}

La satisfacción sexual tiene un papel importante en la vida personal y de pareja (Arrington et al., 2000). Tras el diagnóstico de cáncer de mama y la mastectomía, el interés sexual de la mujer puede verse mermado a causa de los pensamientos que pueden desarrollarse en algunas mujeres (Fleitas-Amaro, 2014). La mastectomía se asocia con serios problemas en el funcionamiento sexual de las pacientes con cáncer de mama. Entre los principales problemas relacionados con la sexualidad que confrontan las mujeres después de la mastectomía, están la sensación de mutilación, los prejuicios relacionados con la autoimagen, la depresión, la consecuente disminución del deseo sexual, la sensación de falta de atractivo sexual, la insatisfacción con el nivel de actividad sexual, las dificultades para alcanzar el orgasmo, la fatiga y el dolor, los cambios en el estado hormonal, los problemas con la sensación en el área del pecho y las dificultades derivadas de la sequedad vaginal (Manganiello et al., 2011).

Las mujeres afectadas por el cáncer de mama pueden mostrar respuestas sexuales diferentes, con reacciones que van desde la fatiga y el cansancio, hasta el dolor. Tras la quimioterapia, resulta frecuente la pérdida del apetito sexual, así como la sequedad vaginal, el dolor durante el coito y la dificultad para alcanzar el orgasmo. Estos factores incrementan las inseguridades sexuales y afectivas debidas a los cambios físicos (cicatriz de mastectomía, linfoedema, caída del cabello, etc.) y emocionales (ansiedad, depresión, angustia).

Es importante destacar que las implicaciones en la sexualidad de la paciente también dependen del tipo de mastectomía por el que haya pasado (Rincón et al., 2012). Existe un mayor funcionamiento sexual en las pacientes con mastectomía simple, debido a que la cirugía trae consigo una menor sintomatología. Esto puede explicar que, cuanto mayor sea la molestia en el postoperatorio, peor será el funcionamiento sexual en la mujer.

La calidad de las relaciones sexuales es otro aspecto importante que también se ve afectado después de la mastectomía. Así lo demostró Gardikiotis et al. (2015) cuando informa de que el $25 \%$ de las participantes en su estudio afirman no estar satisfechas durante las relaciones sexuales ni con el nivel de deseo sexual. López-Cuevas et al. (2008) informaron que el $71 \%$ de sus entrevistadas reportaron un deterioro en su capacidad de obtener placer sexual y el $48 \%$ sintieron disminución del placer obtenido a través de las caricias. Se considera que estos cambios están asociados, muy probablemente, a los sentimientos negativos de falta de atractivo y el miedo al rechazo por parte de la pareja, así como a la falta de comunicación adecuada entre ambos cónyuges en lo referente a estos temas íntimos.

\section{Retos en la Relación de Pareja Tras la Mastectomía}

Las investigaciones demuestran que la cirugía de mastectomía puede afectar a las relaciones de pareja tanto de manera positiva como negativa, dependiendo de factores como el nivel de satisfacción que hubiera en la relación antes del cáncer, las sensaciones de la mujer tras la mastectomía y el apoyo recibido durante el proceso. Avci et al. (2009) exponen que las perspectivas individuales de la pareja ante el cáncer y los diversos tratamientos son un factor importante relacionado con el ajuste conyugal en los pacientes. Por ejemplo, un estudio realizado por Manganiello et al. (2011) reveló que entre las mujeres que informaron tener relaciones comprensivas y gratificantes antes de la cirugía, pocas se quejaron de sentir insatisfacción sexual dos años después del tratamiento. Este autor concluye que la actividad y la satisfacción sexual dependen de la capacidad de la 
pareja de apoyarse mutuamente durante la experiencia de la lucha contra la enfermedad.

En los últimos años, Puerto Rico presenta una alta incidencia de cáncer de mama. Durante el periodo entre los años 2006 al 2010, un promedio de 1,766 mujeres al año, recibieron el diagnóstico de cáncer de mama invasivo (Tolero et al., 2013). A pesar de que el cáncer de mama es una enfermedad que lleva siendo estudiada durante muchos años en Puerto Rico, se ha desatendido la necesidad de que estas pacientes sean tratadas de una manera holística. En Puerto Rico, son escasas las investigaciones que se centran en el estudio de la variable de la satisfacción sexual y sus implicaciones en la relación de pareja. Por tanto, en este artículo se presentará un estudio cuantitativo centrado en examinar el impacto de la cirugía de mastectomía en la satisfacción sexual de la mujer en el marco de una relación de pareja. Los objetivos del estudio fueron: (1) evaluar si la cirugía de mastectomía impacta significativamente la satisfacción sexual de las participantes, (2) evaluar si la cirugía de mastectomía impacta la relación de pareja de las participantes, (3) evaluar si la cirugía de mastectomía impacta los síntomas de depresión de las participantes.

\section{MÉTODO}

\section{Diseño de Investigación}

Se realizó un estudio de método cuantitativo de alcance exploratorio con un diseño trasversal, no experimental, de grupo comparativo (mujeres diagnosticadas con cáncer de mama y sometidas a mastectomía versus mujeres sin cáncer ni mastectomía) (Hernández-Sampieri, 2014). El estudio fue por disponibilidad. La información se recogió de manera presencial tipo entrevista y en línea para ambos grupos.

\section{Participantes}

Se reclutaron 60 mujeres, de las cuales fueron 30 diagnosticadas con cáncer de mama y sometidas a mastectomía y 30 sin el diagnóstico de cáncer ni mastectomía. Las participantes podían tener el cáncer activo o en cualquier tipo de remisión. Los criterios de inclusión fueron: (1) ser mujer puertorriqueña, (2) mayor de 21 años, (3) en una relación de pareja, (4) haber pasado por un proceso de mastectomía realizada hace 6 meses o más y (5) la mastectomía haya sido realizada por diagnóstico de cáncer de mama.

Para el grupo comparativo fueron: (1) ser puertorriqueña, (2) mayor de 21 años, (3) en una relación de pareja, (4) no haber sido diagnosticada con cáncer. Los criterios de exclusión fueron: (1) haberse realizado una mastectomía como método preventivo, (2) padecer una condición psiquiátrica severa. Para el grupo comparativo fueron: (1) padecer alguna enfermedad crónica, (2) padecer algún tipo de cáncer, y (3) padecer una condición psiquiátrica severa.

\section{Instrumentos}

Se utilizaron cuatro instrumentos, estos fueron administrados a ambos grupos por igual. No obstante, el instrumento de datos sociodemográficos y sexuales fue diferente para ambos grupos.

Cuestionario de Datos Sociodemográficos y Sexualidad. Este cuestionario fue desarrollado por la investigadora principal. Para el grupo con cáncer, se exploraron los datos personales e información sociodemográfica de las participantes, además información sobre actividad sexual y relacionada al tiempo transcurrido desde la mastectomía y otros tratamientos experimentados por la encuestada. El cuestionario del grupo comparativo exploró información demográfica e información sobre actividad sexual y relación de pareja, pero no incluyó preguntas relacionadas al cáncer.

\section{Escala de Satisfacción Sexual Subjetiva (ESSS).} Este instrumento fue desarrollado por González-Rivera et al. (2017), el mismo tiene el objetivo de medir el constructo de satisfacción sexual bajo cuatro dimensiones identificadas en la literatura: valoración subjetiva, aspecto emocional, ejecución sexual y autoimagen. El mismo consta de 20 reactivos, con una escala de respuesta de cuatro puntos que va desde (1) totalmente en desacuerdo hasta (4) totalmente de acuerdo. El puntaje total de la escala varía entre 20 a 80 puntos; a mayor puntuación obtenida, mayor satisfacción sexual exhibe la persona. La ESSS cuenta con un alpha de Cronbach de .91 lo que implica una consistencia interna excelente.

Escala de Satisfacción en la Pareja (ESP-10). Esta escala fue desarrollada por González-Rivera y VerayAlicea (2018), y su propósito es medir la satisfacción en la pareja de acuerdo con el modelo unidimensional. En esta escala la satisfacción de pareja es definida como una autoevaluación positiva o negativa que la persona realiza sobre su relación de pareja actual 
(González-Rivera \& Veray-Alicea, 2018). La ESP-10 está conformada por 10 reactivos, en una escala de respuesta de cuatro puntos que va desde (1) totalmente en desacuerdo a (4) totalmente de acuerdo. Las puntuaciones obtenidas se pueden encontrar entre 10 y 40; a mayor puntuación obtenida se entiende que hay mayor satisfacción de pareja. Esta escala cuenta con un alpha de Cronbach de .91 lo que sugiere una consistencia interna excelente.

\section{Cuestionario sobre la Salud del Paciente, Escala} de Depresión-8 (PHQ-8). Este cuestionario fue desarrollado por Kroenke et al. (2009) con el propósito de evaluar, diagnosticar, monitorear y medir la gravedad de la depresión según estipula el Manual diagnóstico y estadístico de los trastornos mentales en su cuarta edición (DSM-IV). Este cuestionario cuenta con 8 reactivos, la escala de respuesta se distribuye de la siguiente manera: (0-1 día) nunca, (2-6 días) algunos días, (7-11 días) más de la mitad de los días y (1214 días) casi todos los días. Las puntuaciones van de 0 a 3, asignado a cada categoría respectivamente. La puntuación total obtenida puede variar entre los valores de 0 y 24 puntos. A mayor puntuación, mayor es la severidad de los síntomas depresivos. Este cuestionario cuenta con un alpha de Cronback de .88 lo que implica una consistencia interna buena.

\section{Procedimientos Generales}

Una vez la propuesta de investigación fue aprobada por el Comité Institucional de Revisión (IRB) de la Ponce Health Sciences University (\#1905014284), se procedió a reclutar la muestra. El procedimiento de reclutamiento se llevó a cabo de manera presencial y en línea. Para el reclutamiento presencial, se distribuyeron unos volantes de promoción en la comunidad. Las mujeres interesadas contactaron a la investigadora principal, se les proporcionó información sobre el estudio y concretó una cita según la disponibilidad de la participante. Se compartió el volante de promoción a través de correo electrónico, grupos de apoyo para mujeres con cáncer y redes sociales tales como: Facebook, Instagram y WhatsApp. El volante de promoción tenía adjunto un enlace al cual las participantes podían acceder a la investigación a través de la plataforma de LimeSurvey y también contaba con un correo electrónico para aquellas mujeres que deseaban participar mediante entrevista. Una vez la persona decidía participar, se le ofrecía una hoja de consentimiento informado, y luego de aceptada y firmada (presencial o electrónicamente) completaban una hoja con datos sociodemográficos y sexuales y los tres instrumentos del estudio. El proceso tomó alrededor de 15 a 30 minutos para completarlo. Para garantizar la protección y anonimato en los datos de las participantes, el nombre de las participantes no fue incluido en los documentos.

\section{Análisis de Datos}

La data obtenida se analizó utilizando Statistical Package for the Social Sciences (SPSS) Versión 26.0. Para los datos sociodemográficos y sexuales se utilizaron estadísticas descriptivas tales como: frecuencias, porcientos y promedios. Para los tres objetivos del estudio se llevó a cabo una prueba de Levene para determinar la igualdad en las varianzas. Debido a que la prueba de Levene solo encontró igualdad en las varianzas para la escala de satisfacción de pareja $(F=$ $10.059, p=.002)$ y desigualdad para satisfacción sexual $(F=3.620, p=.062)$ y depresión $(F=3.075, p=$ .085), se llevó a cabo un análisis de prueba $U$ de Mann-Whitney de muestras independientes para la comparación de los grupos. Los análisis post hoc muestran que hubo un gran tamaño del efecto $(f=.35)$, por lo que el estudio debe repetirse con una muestra más grande.

\section{RESULTADOS}

\section{Datos Sociodemográficos de la Muestra}

La muestra total constó de 60 mujeres $(n=60)$ autoidentificadas como del género femenino. Esta muestra se subdividió en dos grupos, el grupo de mujeres con cáncer $(n=30)$ quienes fueron mujeres diagnosticadas con cáncer de mama sometidas a mastectomía y el grupo comparativo quienes fueron mujeres que nunca han tenido el diagnóstico de cáncer $(n=$ 30). Se eliminó una participante por omisión de la mayoría de los reactivos, resultando en 29 participantes para el grupo con cáncer. Para aquellas participantes que omitieron uno o dos reactivos, se procedió a promediar los espacios en blanco. Las participantes se encontraban entre las edades de 22 y 77 años, con un promedio de 44 años.

Al explorar el estado civil de las participantes, los resultados indican que la mayoría estaban casadas $(f$ $=32,54.2 \%)$, el $32.2 \%(f=19)$ tenían pareja, pero no convivían, mientras que el $13.6 \%(f=8)$ convivía con 
su pareja. Por otra parte, el $96.6 \%(f=57)$ de la muestra mantenía una relación con una persona del sexo opuesto y el $3.2 \%(f=2)$ mantenía una relación con una persona del mismo sexo al momento de participar en el estudio. Al explorar el tiempo de relación que mantenían las participantes con sus parejas, se obtiene un promedio de 156 meses, lo que es equivalente a 13 años de relación. Al hacer una comparación entre los grupos, se observan diferencias significativas en el tiempo de relación entre ambos grupos. Estos resultados revelan que la mayoría de las participantes del grupo con cáncer tenían de 11 a 15 años en una relación de pareja. A diferencia de la mayoría de las participantes del grupo comparativo quienes reportaron un tiempo de relación entre dos a diez años.

Al contemplar la cantidad de tiempo desde que las participantes del grupo experimental le realizaron su primera mastectomía, los datos presentan una media de 91.66 meses (7.64 años). De este grupo, solo una participante $(f=1)$ se realizó una segunda mastectomía la cual fue hace 22 años. Los resultandos también revelaron que la mayoría de las participantes $(58.6 \%$, $f=17$ ) no fue orientada sobre los efectos de la mastectomía en su vida sexual. Se exploró si las participantes recibieron otro tratamiento oncológico adicional a la mastectomía y el 79.3\% ( $f=23)$ del grupo con cáncer indicó que sí.

Del grupo experimental, el $25.4 \%$ de las participantes $(f=15)$ había atravesado el proceso de reconstrucción mamaria, y de ellas, el 100\% se realizó implante. El 11.9\% $(f=7)$ tenía una prótesis mamaria. Al explorar el tipo de prótesis mamaria, el 1.7\% $(f=1)$ indicó utilizar expansor, $1.7 \%(f=1)$ gel, $1.7 \%(f=1)$ silicón y el 1.7\% $(f=1)$ sostén. Luego de la mastectomía, el $11.9 \%(f=7)$ de las participantes del grupo experimental se realizaron un tatuaje simulando el pezón en el área del seno luego de la mastectomía.

En cuanto a la relación de pareja, la mayoría de las participantes del grupo experimental describen una buena relación con sus parejas antes de la mastectomía $(65.5 \%, f=19)$. El 24.1\% $(f=7)$ la describen como regular, mientras que el $6.9 \%(f=2)$ la describen como distante y el $3.4 \%(f=1)$ la describe como conflictiva. $\mathrm{Al}$ explorar si la pareja de las participantes del grupo experimental ha sido de apoyo antes y después de la mastectomía, la mayoría de las participantes $(f=15$, $51.7 \%)$ mencionó que estaba totalmente de acuerdo con esta premisa. El 6.9\% $(f=2)$ de las participantes mencionó estar de acuerdo con la premisa, mientras que el $20.6 \%(f=6)$ mostró estar en desacuerdo y el $20.6 \%(f=6)$ mostró estar totalmente en desacuerdo.

Al explorar la actividad sexual, el 89.8\% $(f=53)$ de las participantes de la muestra total se encuentra sexualmente activa y el $10.2 \%(f=6)$ no está sexualmente activa. De la muestra total, el $93.2 \%$ no mantiene relaciones de pareja adicionales a su pareja principal. Se les pidió a las participantes que clasificaran su vida sexual antes de la mastectomía, a esto el $48.2 \%$ $(f=14)$ de las participantes del grupo con cáncer indicaron que esta era satisfactoria y el $37.9 \%(f=11)$ indicó que esta era muy satisfactoria. Por otra parte, el $10.3 \%(f=3)$ indicó que sus relaciones sexuales previas a la mastectomía eran poco satisfactorias y el $3.4 \%(f=1)$ indicó que eran nada satisfactorias.

Al medir la actividad sexual del grupo experimental luego de la mastectomía, se observa una disminución de la práctica en la mayoría de las participantes. El 55.1\% $(f=16)$ reportó una disminución en la actividad sexual luego de la mastectomía. Los resultados indican que la media de la actividad sexual en un mes fue de seis veces $(M=6.66)$. El 50\% $(f=30)$ de las participantes reportaron tener de ninguno a cinco encuentros sexuales al mes, mientras que el 32\% $(f=19)$ indicaron tener relaciones sexuales de seis a 10 veces en el mes. El 17\% $(f=10)$ de las participantes indican tener relaciones sexuales de 11 a 25 veces al mes. Al comparar la actividad sexual del grupo con cáncer con la del grupo comparativo se observa que ambos grupos refieren mantener una frecuencia de cuatro veces al mes.

\section{Análisis de los Objetivos}

El primer objetivo de esta investigación era evaluar si la cirugía de mastectomía impactaba significativamente la satisfacción sexual de la mujer con cáncer. Al llevar a cabo un análisis de prueba U de MannWhitney de muestras independientes entre el grupo con cáncer compuesto por mujeres con cáncer de seno y sometidas a mastectomía y el grupo comparativo compuesto por mujeres sin cáncer ni mastectomía, se encontraron diferencias significativas en la satisfacción sexual $U\left[N_{\text {cáncer }}=29, N_{\text {no-cancer }}=30\right)=587.500, z=$ 2.931, $p=.003$ ], siendo menor en el grupo con cáncer (Ver Tabla 1). 
Tabla 1

Pruebas U de Mann-Whitney de muestras independientes para ESSS, ESP-10 y PHQ8.

\begin{tabular}{lcccccc}
\hline Instrumento & $\begin{array}{c}\text { Mann-Whit- } \\
\text { ney U }\end{array}$ & $\begin{array}{c}\text { Wilcoxon } \\
\text { W }\end{array}$ & $\begin{array}{c}\text { Test Statis- } \\
\text { tics }\end{array}$ & $\begin{array}{c}\text { Standard } \\
\text { Error }\end{array}$ & $\begin{array}{c}\text { Standarized } \\
\text { Test Statistic }\end{array}$ & $\begin{array}{c}\text { Sig } \\
\text { ESSS }\end{array}$ \\
\hline 587.500 & 1052.500 & 587.500 & 62.262 & 2.931 & $.003^{* *}$ \\
Valoración Subjetiva & 617.00 & 1082.00 & 617.00 & 65.851 & 2.536 & $.011^{*}$ \\
Aspecto Emocional & 655.00 & 1120.00 & 655.00 & 66.723 & 3.072 & $.002^{* *}$ \\
Ejecución Sexual & 448.500 & 913.500 & 448.500 & 66.829 & -.022 & .982 \\
Autoimagen & 533.00 & 998.00 & 533.00 & 61.616 & 2.077 & $.038^{*}$ \\
ESP & 654.500 & 119.500 & 654.500 & 64.093 & 3.659 & $.000^{* * *}$ \\
PHQ8 & 527.000 & 992.000 & 527.000 & 66.687 & 1.155 & .248 \\
\hline
\end{tabular}

El segundo objetivo de esta investigación era evaluar si la cirugía de mastectomía impactaba significativamente la relación de pareja del grupo con cáncer. Al llevar a cabo un análisis de prueba U de MannWhitney de muestras independientes se encontraron diferencias significativas en la satisfacción de pareja $U\left[N_{\text {cáncer }}=29, N_{\text {no-cancer }}=30\right)=654.500, \mathrm{z}=3.659, p<$ .000], siendo menor en el grupo con cáncer.

El tercer objetivo de esta investigación era evaluar si la cirugía de mastectomía impactaba significativamente los síntomas de depresión del grupo con cáncer. Al llevar a cabo un análisis de prueba U de MannWhitney de muestras independientes no se encontraron diferencias significativas entre los grupos $U\left[N_{\text {cán- }}\right.$ cer $\left.=29, N_{\text {no-cancer }}=30\right)=527, \mathrm{z}=1.155, p=.248$ ].

\section{DISCUSIÓN}

El presente estudio tuvo como finalidad examinar el impacto de la cirugía de mastectomía en la satisfacción sexual, satisfacción de pareja y síntomas de depresión en mujeres sobrevivientes de cáncer de seno con pareja. El primer objetivo fue explorar si existían diferencias significativas entre la satisfacción sexual de las mujeres sometidas a mastectomía y las mujeres del grupo comparativo. Los resultados evidenciaron diferencias significativas en las muestras, lo cual sugiere que las mujeres con cáncer de seno y sometidas a mastectomía, reportaron menor satisfacción sexual que las mujeres sin cáncer. Estos resultados también revelaron diferencias significativas en las escalas que evaluaban valoración subjetiva, autocuidado y aspectos emocionales de la sexualidad, lo que podría significar que estas mujeres experimentan y perciben su sexualidad de forma insatisfactoria. Sin embargo, los resultados no demostraron diferencias significativas en la escala que evalúa ejecución sexual. De ahí se infiere que estas mujeres parecen estar satisfechas con su autopercepción y desempeño sexual. No obstante, estas diferencias también podrían implicar dificultades con la pareja, así como conflictos psicológicos relacionados con el momento del acto sexual. La satisfacción sexual puede ser resultado de la interacción de numerosos factores. Por consiguiente, los resultados expuestos anteriormente podrían concordar con los estudios que asocian la cirugía de mastectomía a problemas en el funcionamiento sexual (Manganiello et al., 2011).

El segundo objetivo fue analizar si la cirugía de mastectomía impactaba significativamente en la relación de pareja de las mujeres con cáncer. Los resultados evidenciaron diferencias significativas. Esto sugiere que las mujeres sometidas a mastectomía pudiesen estar menos satisfechas con su relación de pareja que las mujeres sin cáncer. Los resultados coinciden con varios autores que sostienen que este proceso quirúrgico sí afecta negativamente a las parejas, provocando un distanciamiento emocional entre los cónyuges, la redefinición de los roles dentro de la pareja (Hawkins et al., 2009), cambios negativos en las relaciones sexuales (Rowland \& Metcalfe, 2014), la posible separación conyugal e incluso la tolerancia hacia relaciones extramatrimoniales (Airaldi, 2010). Sin embargo, otros estudios contrastan los resultados de esta investigación, demostrando que la relación con su pareja era más cercana en la actualidad (Vázquez et al., 2009), valoraban más la vida de la mujer que la pérdida del seno (Hoga et al., 2008), gozaban mayor cercanía emocional y se sentían muy cercanas a sus compañeros después de la mastectomía (Airaldi, 2010).

Por último, el tercer objetivo fue analizar si la cirugía de mastectomía impactaba significativamente en los síntomas de depresión de las participantes. Los resultados evidenciaron que no había diferencias significativas en esta muestra. Sin embargo, la literatura 
ha sugerido lo contrario. Por ejemplo, Kocan y Gursoy (2016) y Manganiello et al. (2011) afirman que la mastectomía impacta negativamente en la sexualidad de la mujer y esto se asocia a una serie de problemas físicos y psicológicos tales como la depresión. Sin embargo, estudios en Puerto Rico como el de CrespoFernández y Rivera-García (2012) han encontrado que las mujeres puertorriqueñas que sobrevivieron el cáncer de mama, mostraron una alta capacidad de resiliencia en donde lucharon por superarse, desarrollaron fortalezas cognoscitivas y emocionales durante la recuperación, hallaron el propósito y los aspectos positivos en la vida y vieron la enfermedad como una oportunidad para crecer y beneficiarse.

\section{Implicaciones}

Las secuelas psicológicas de la mastectomía y los tratamientos contra el cáncer de mama pueden afectar de forma directa las relaciones afectivosexuales de las mujeres que padecen esta enfermedad. La mayoría de las investigaciones se han reducido al ámbito psicológico como al de la relación de pareja, sin embargo, el presente estudio, ha sido de los primeros en evaluar las implicaciones del cáncer en la sexualidad y relación de pareja de estas mujeres. Los hallazgos de este estudio contribuyen a aumentar la información disponible en el tema, particularmente en el contexto de la cultura puertorriqueña. Además, permite que se genere mayor interés en el área de la sexualidad de la mujer con cáncer de mama. Los hallazgos de este estudio abren espacio a varias interrogantes cuando se comparan con estudios realizados con muestra estadounidense, por ejemplo, permite cuestionar el rol que tiene la resiliencia en las relaciones afectivosexuales de las mujeres a quienes le han realizado una mastectomía. Asimismo, permite interrogarse cuáles son aquellas expectativas socioculturales que juegan un rol en la autoevaluación de la satisfacción sexual y de pareja. Por tanto, estos datos abren paso a un amplio tema de conocimiento del que aún se desconoce mucho.

En cuanto al aspecto clínico, estos datos proveen información importante para fines de intervención terapéutica. Por ejemplo, visibiliza los factores que pueden influir en la insatisfacción en la relación de pareja en mujeres que han atravesado una mastectomía. Por tanto, facilita que los profesionales de la psicología exploren estos aspectos con sus pacientes. También, evidencia la importancia de la integración de profesionales de psicología en ambientes médiconcológicos y crea consciencia sobre la importancia de que los profesionales de la psicología estén preparados para abordar temas de sexualidad y relaciones de pareja ya que claramente juegan un rol importante en el bienestar integral de las mujeres que han sido sujetas a mastectomía. Por último, visibiliza la necesidad de que las parejas de las mujeres que han tenido mastectomías puedan integrarse en procesos de terapia y/o consejería psicológica.

\section{Limitaciones del Estudio}

$\mathrm{Al}$ realizar investigaciones es difícil mantener bajo control todos los factores que puedan impactar sobre los resultados. La presente investigación no está exenta de esta situación, por lo que a continuación se presentan las siguientes limitaciones: (1) el tamaño de la muestra fue pequeño (el mínimo sugerido luego de los cálculos en el programa $\mathrm{G}^{*}$ Power) y por disponibilidad lo cual impide generalizar los resultados a la población puertorriqueña y disminuye el poder estadístico, (2) los perfiles sociodemográficos de la muestra como edad y tiempo en la relación no fueron homogéneos entre los grupos, y (3) no existía estudios previos sobre las implicaciones de la mastectomía en aspectos sexuales o de pareja de la mujer con cáncer de seno y sometida a mastectomía en Puerto Rico sobre los que asentar la investigación.

\section{Recomendaciones}

A la luz de los resultados obtenidos en la presente investigación se recomienda lo siguiente: (1) el desarrollo de más investigaciones en Puerto Rico que faciliten el conocimiento y entendimiento sobre las implicaciones del cáncer de mama y la mastectomía en la vida sexual y de pareja de la mujer, (2) replicar este estudio con una población más grande, homogénea y aleatoria, (3) la creación de un instrumento que evalúe detalladamente los cambios afectivosexuales específicos en la mujer tras la mastectomía, (4) incorporar la percepción de la pareja dentro de las entrevistas, (4) concientizar entre los profesionales de la salud sobre la importancia de profesionales de la psicología en el ambiente mediconcológico y la importancia de la terapia psicológica (individual y de pareja) durante el proceso de tratamiento oncológico (5) ofrecer intervenciones dirigidas a la psicoeducación sobre los efectos de la mastectomía en la sexualidad y la pareja. 


\section{CONCLUSIÓN}

Este estudio examinó el impacto de la cirugía de mastectomía en la satisfacción sexual, satisfacción de pareja y síntomas de depresión en mujeres sobrevivientes de cáncer de seno con pareja. Los hallazgos señalan que existen diferencias significativas en la satisfacción entre mujeres con cáncer de mama y sometidas a mastectomía en comparación con el grupo de comparación. También se observaron diferencias significativas entre estos grupos en cuanto a la satisfacción de pareja. No obstante, la cirugía de mastectomía no mostró impactar de forma significativa los síntomas de depresión de las mujeres participantes. Los hallazgos de esta investigación brindan el espacio para el continuo desarrollo del tema a través de la investigación, y hacen un llamado a los profesionales de la psicología a estar conscientes de problemáticas relacionadas a la sexualidad y satisfacción de pareja en mujeres que han tenido una mastectomía.

Financiamiento: La presente investigación no fue financiada por alguna entidad ni patrocinador.

Conflicto de Intereses: No existen conflictos de intereses de parte de los autores de la investigación.

Aprobación de la Junta Institucional para la Protección de Seres Humano en la Investigación: Comité Institucional de Revisión (IRB) de la Ponce Health Sciences University (\#1905014284).

Consentimiento o Asentimiento Informado: Todo participante completó un consentimiento informado.

Proceso de Revisión: Este estudio ha sido revisado por pares externos en modalidad de doble ciego.

\section{REFERENCIAS}

Airaldi, M. C. (2010). Sexualidad y relaciones de pareja en mujeres mastectomizadas de una muestra paraguaya. Eureka, 7(1), 143162. https://psicoeureka.com.py/sites/default/files/articulos/eureka-7-1-10-14.pdf

Andrzejczak, E., Markocka-Ma,czka, K., \& Lewandowski, A. (2013). Partner relationships after mastectomy in women not offered breast reconstruction. Psycho-Oncology, 22(7), 1653-1657. https://doi.org/10.1002/pon.3197

Avci, I. A., Okanli, A., Karabulutlu, E., \& Bilgili, N. (2009). Women's marital adjustment and hopelessness levels after mastectomy. European Journal of Oncology Nursing, 13(4), 299-303. https://doi.org/10.1016/j.ejon.2009.03.011

Crespo-Fernández, L., \& Rivera-García, M. (2012). El poder de la resiliencia generado por el cáncer de mama en mujeres en
Puerto Rico. Revista Puertorriqueña de Psicología, 23(1), 109-126. http://www.ojs.repsasppr.net/index.php/reps/article/view/201/201

Faghani, S., \& Ghaffari, F. (2016). Effects of sexual rehabilitation using the PLISSIT Model on quality of sexual life and sexual functioning in post-mastectomy breast cancer survivors. Asian Pacific Journal of Cancer Prevention, 17(11), 4845-4851. http://doi.org/10.22034/APJCP.2016.17.11.4845

Fleitas-Amaro, M. M. (2014). Cáncer de mama: mastectomía y sexualidad. https://sifp.psico.edu.uy/sites/default/files/Trabajos\%20finales/\%20Archivos/TFG\%20Melisa\%20Fleitas.pdf

Freysteinson, W. M., Deutsch, A. S., Davin, K., Lewis, C., Sisk, A., Sweeney, L., Wuest, L., \& Cesario, S. K. (2015). The mirror program: Preparing women for the postoperative mastectomy mirror-viewing experience. Nursing Forum, 50(4), 252-257. http://doi.org/10.1111/nuf.12108

Gardikiotis, I., Iorga, M., \& Sztankovszky, L. Z. (2015). Sexuality, body image and psychological well-being of patients with mastectomy after breast cancer. Journal of Surgical Oncology, 119(3), 849-855.

González-Rivera, J. \& Veray-Alicea, J. (2018) Propiedades psicométricas de la Escala de Satisfacción en la Pareja en una muestra de adultos puertorriqueños. Revista Electrónica de Psicología Iztacala, 21(1), 111-119. http://www.revistas.unam.mx/index.php/repi/article/view/64019/56188

González-Rivera, J., Veray-Alicea, J., Santiago-Santos, D., CastroCastro, S., \& Quiñones-Soto, R. (2017) Desarrollo y validación de una escala para medir satisfacción sexual subjetiva en adultos puertorriqueños. Salud y Conducta Humana, 4(1), 52-63. https://static1.squarespace.com/static/50c39c53e4b097533b349 2dd/t/59ecc668a803bb707b4d1dab/1508689514828/GonzálezRivera\%2C+2017+\%282\%29.pdf

Hawkins, Y., Ussher, J., Gilbert, E., Perz, J., Sandoval, M., \& Sundquist, K. (2009). Changes in sexuality and intimacy after the diagnosis and treatment of cancer: The experience of partners in a sexual relationship with a person with cancer. Cancer nursing, 32(4), 271-280. https://doi.org/10.1097/ncc.0b013e31819b5a93

Hernández-Sampieri, R., Fernández-Collado, C., \& Baptista-Lucio, P. (2014). Metodología de la investigación (6th ed.). McGraw Hill.

Hoga, L. A. K., Mello, D. S., \& Días, A. F. (2008). Psychosocial perspectives of the partners of breast cancer patients treated with a mastectomy: An analysis of personal narratives. Cancer Nursing, 31(4), 318-325. http://doi.org/10.1097/01.NCC.0000305748.43367.1b

Hungr, C., Sánchez-Varela, V., \& Bober, S. L. (2017). Self-image and sexuality issues among young women with breast cancer: practical recommendations. Revista de Investigación Clínica, 69(2), 114-122. https://doi.org/10.24875/ric.17002200

Instituto Nacional del Cáncer. (2015). ¿Qué es el cáncer? https://www.cancer.gov/espanol/cancer/naturaleza/que-es

Kroenke, K., Strine, T. W., Spritzer, R. L., Williams, J. B., Berry, J. T. \& Mokdad, A. H. (2009) The PHQ-8 as a measure of current depression in the general population. Journal of Affective 
Disorders, 114(1-3), 163-73.

https://doi.org/10.1016/j.jad.2008.06.026

Linden, W., Vodermaier, A., MacKenzie, R., \& Greig, D. (2012). Anxiety and depression after cancer diagnosis: Prevalence rates by cancer type, gender, and age. Journal of Affective Disorders, 141(2-3), 343-351. https://doi.org/10.1016/j.jad.2012.03.025

López-Cueva, Z. C., González-Ortega, J. M., Gómez-Hernández, M. M., \& Morales-Wong, M. M. (2008). Impacto psicológico y social de la mastectomía en pacientes operadas de cáncer de mama. Revista Médica Electrónica, 30(2). http://www.revmedicaelectronica.sld.cu/index.php/rme/article/view/484/pdf

Manganiello, A., Hoga, L. A. K., Reberte, L. M., Miranda, C. M., \& Rocha, C. A. M. (2011). Sexuality and quality of life of breast cancer patients post mastectomy. European Journal of Oncology Nursing, 15(2), 167-172. http://doi.org/10.1016/j.ejon.2010.07.008
Mckean, L. N., Newman, E. F., \& Adair, P. (2013). Feeling like me again: A grounded theory of the role of breast reconstruction surgery in self-image. European Journal of Cancer Care, 22(4), 493-502. https://doi.org/10.1111/ecc.12055

Rincón-Fernández, M. E., Pérez-San Gregorio, M. A., Borda-Mas, M., \& Martín-Rodríguez, A. (2012). The impact of breast reconstruction on self-esteem and body image in patients with breast cancer. Universitas Psychologica, 11(1), 25-41. http://www.scielo.org.co/pdf/rups/v11n1/v11n1a03.pdf

Rowland, E., \& Metcalfe, A. (2014). A systematic review of men's experiences of their partner's mastectomy: Coping with altered bodies. Psycho-Oncology, 23(9), 963-974. https://doi.org/10.1002/pon.3556

Vázquez-Ortiz, J., Antequera, R., \& Picabia, A. B. (2010). Ajuste sexual e imagen corporal en mujeres mastectomizadas por cáncer de mama. Psicooncología, 7(2-3), 433-451. https://revistas.ucm.es/index.php/PSIC/article/view/PSIC1010220433A

Obra bajo licencia de Creative Commons Atribución 4.0 Internacional (CC BY 4.0).

(C) 2021 Autores. 